\title{
Efficacy of Edaravone in Combination with Dihydropyridine Calcium Ion Antagonist on Hypertensive Intracerebral Hemorrhage
}

\author{
YANG LI, XIU-QIN WU1 ${ }^{1}$, JIA-ZE LI², FU-SHUN WANG ${ }^{3}$, JIAN-ZHONG LI ${ }^{3}$ AND QI FAN ${ }^{*}$ \\ Psychological Center, School of Management, Nanjing Forest Police College, Nanjing, Jiangsu 210023, ${ }^{1}$ Nanjing Institute \\ of Railway Technology, Nanjing, Jiangsu 210031, ${ }^{2}$ school Of Foreign Languages, Nanjing Agricultural University, Nanjing, \\ Jiangsu210026, ${ }^{3}$ School of Psychology, Nanjing University of Chinese Medicine, Nanjing, Jiangsu210023, ${ }^{4}$ Institute of Mental \\ Health, NanJing XiaoZhuang University, Nanjing, Jiangsu, P.R.China, 210017.
}

\section{Li et al: Edaravone in Combination with Dihydropyridine Calcium Ion Antagonist}

\begin{abstract}
This research article planned to investigate the efficacy of edaravone in combination with dihydropyridine calcium ion antagonist on hypertensive intracerebral hemorrhage. Between February 2017 and January 2019, we collected the clinical data from 126 hypertensive intracerebral hemorrhage patients and divided them into the control group and observation group, with 63 patients in each group. Patients in two groups all underwent the regular treatment, while those in the observation group took edaravone in combination with dihydropyridine calcium ion antagonist (nimodipine) for treatment. Following the treatment, we compared the clinical efficacy, plasmin endothelin 1, volume of edema and hematoma and National Institute of Health stroke scale scores. In the control group, the total effectiveness rate of treatment was $71.44 \%$, significantly lower than that of the observation group $(p<0.05)$; following treatment, the level of endothelin 1 in plasma and the volumes of edema and hematoma were all decreased as compared to those before treatment and the decrease in the observation group was more evident $(p<0.01)$. Before treatment, difference in the National Institute of Health stroke scale scores between the control group and the observation group was not evident ( $>>0.05)$, while after 2 and $4 \mathrm{w}$ of treatment, patients in the observation group had scores of $(12.43 \pm 4.07)$ and $(7.52 \pm 1.84)$ points, significantly higher than those in the control group $(\mathbf{p}<\mathbf{0 . 0 1})$. Combined medication of edaravone and dihydropyridine calcium ion antagonist can benefit the patients with promising efficacy and improvement in nervous function.
\end{abstract}

Key words: Edaravone, intracranial hemorrhage, hypertensive, stroke, fibrinolysin.

Intracerebral hemorrhage refers to the non-traumatic, primary, brain parenchymal hemorrhage that is usually caused by the arteriolar sclerosis and complication of hypertension, so it is also known as hypertensive intracerebral hemorrhage $(\mathrm{HICH})^{[1]}$. The attack of $\mathrm{HICH}$ can induce the intracerebral hematoma and edema that can injure or suppress the brain tissues, thereby triggering the nerve dysfunction and other symptoms. Due to the high prevalence, sudden attack and rapid progression, $\mathrm{HICH}$ is usually featured by the high morbidity rate and death rate. Clinically, it is believed that secondary brain edema is one of the risk factors that contribute to the deterioration of condition: After cerebral hemorrhage, hematoma suppresses the peripheral nerves, which, plus the inflammation of the damage vessels and nerves, would facilitate the generation of free radicals and induce the edema surrounding the bleeding site, thereby aggravating the disease ${ }^{[2,3]}$. At present, $\mathrm{HICH}$ patients are mainly treated by the medication, including edaravone as the potent eliminator of free radicals and dihydropyridine calcium ion antagonist (nimodipine) for vasodilation and mitigating the cerebral edema ${ }^{[4,5]}$. In this study, we enrolled 126 patients as subjects, aiming to clarify the effect of the combined medication of edaravone and nimodipine on $\mathrm{HICH}$ patients.

Between February 2017 and January 2019, we enrolled a total of $126 \mathrm{HICH}$ patients who were admitted to this hospital for treatment to analyze their clinical data, among which patients with hypertensive history, intracerebral hemorrhage confirmed by heat Computed tomography and admission within $24 \mathrm{~h}$ after onset were

*Address for correspondence

E-mail: kangzou12@163.com 
enrolled, while those with severe dysfunction in liver or kidney, or with traumatic hematoma were excluded. These patients were divided into two groups, control group and observation group, with 63 patients in each group. In the control group, there were 37 males and 26 females, aged between 51 and 72 y old, with an average of $(59.78 \pm 5.27) \mathrm{y}$; for hemorrhage site, there were 26 patients with hemorrhage in basal ganglia, 11 in lobe, 16 in thalamus and 10 in cerebellum. In the observation group, there were 38 males and 25 females, aged between 52 and 73 y old, with an average of (59. 85 \pm 5.31$) \mathrm{y}$; for hemorrhage site, there were 25 patients with hemorrhage in basal ganglia, 9 in lobe, 18 in thalamus and 12 in cerebellum. Differences in the baseline data showed no statistical significance between two groups ( $>0.05)$.

Patients in the two groups underwent the regular comprehensive treatment, including oxygen inhalation, medication of mannitol to control the intracerebral pressure, sustaining the water-electrolyte balance, anti-inflammation and protecting the brain cells. In observation group, patients would additionally take $30 \mathrm{mg}$ edaravone via intravenous infusion (Xi'an Lijun Pharmaceutical Co., Ltd, SFDA Approval No.: H20120042, $20 \mathrm{~mL}: 30 \mathrm{mg}$ ), twice a day for $14 \mathrm{~d}$, besides, $10 \mathrm{mg}$ nimodipine (Shandong Xinghua Pharmaceutical Co., Ltd, SFDA Approval No.: H10950226, 2 mg: 10 $\mathrm{mL}$ ) was given via intravenous infusion in $500 \mathrm{~mL} 5$ $\%$ glucose solution, once per day, for $10 \mathrm{~d}$, which was then changed into the oral administration of $40 \mathrm{mg}$ nimodipine (Yabao Pharmaceutical Group Co., Ltd; SFDA Approval No.: H14022821, $20 \mathrm{mg} /$ tablet), three times a day, for $30 \mathrm{~d}$.

Clinical efficacy was compared between two groups as per the following criteria: Excellence for decrease in edema volume by $10 \mathrm{~mL}$ or more, in National Institute of Health stroke scale (NIHSS) scores by $75 \%$ or more; improvement for decrease in edema volume by 5 to $9 \mathrm{~mL}$, and in NIHSS score by $25 \%$ to $74 \%$; failure for decrease in NIHSS score by less than $25 \%$, and no improvement in the mental status, consciousness or hematoma. Total effectiveness was the total cases of excellence and improvement. We compared the level of endothelin 1 (ET1) in plasma, edema and hematoma volume and NIHSS scores between two groups. NIHSS scale included the following items: consciousness, limb motion, gaze and facioplegia, with a score ranging from 0 to 42 points.

SPSS 17.0 software was used for data analysis in this study. Measurement data in normal distribution were presented by mean \pm standard deviation, and compared between two groups by t test. Enumeration data were presented by $\mathrm{n}(\%)$, and compared by chi-square test. $\mathrm{p}<0.05$ suggested that the difference had statistical significance.

In the control group, the total effectiveness rate was significantly lower than that in the observation group $(\mathrm{p}<0.05$; Table 1$)$.

Following treatment, the level of ET1 in plasma and the volume of edema and hematoma were all decreased in two groups as compared to those before treatment, and the decreases in the observation group were more evident than those in the control group $(\mathrm{p}<0.01$; Table 2).

No significant differences were identified in comparison of the NIHSS scores before treatment between two groups ( $p>0.05$ ), while after two or four w of treatment, patients in the observation group gained a higher NIHSS score than that in the control group $(\mathrm{p}<0.05$; Table 3$)$.

TABLE 1: COMPARISON OF THE CLINICAL EFFICACY BETWEEN TWO GROUPS [N (\%)]

\begin{tabular}{|c|c|c|c|c|c|c|}
\hline \multirow{2}{*}{ Item } & \multicolumn{2}{|c|}{ Control group $(n=63)$} & \multicolumn{2}{|c|}{ Observation group $(n=63)$} & \multirow{2}{*}{$\chi^{2}$} & \multirow{2}{*}{$\mathrm{p}$} \\
\hline & $\mathbf{N}$ & Percentage (\%) & $\mathbf{N}$ & Percentage (\%) & & \\
\hline Failure & 18 & 28.56 & 6 & $9.52 a$ & 6.5783 & $<0.05$ \\
\hline Improvement & 23 & 36.51 & 24 & 38.09 & 0.0347 & $>0.05$ \\
\hline Excellence & 21 & 33.33 & 33 & $52.39 a$ & 3.4926 & $>0.05$ \\
\hline $\begin{array}{l}\text { Total } \\
\text { effectiveness } \\
\text { rate }\end{array}$ & 44 & 71.44 & 57 & $89.48 \mathrm{a}$ & 6.5324 & $<0.05$ \\
\hline
\end{tabular}

Note: ${ }^{a} p<0.05$ represents the significant difference in comparison between groups. 
www.ijpsonline.com

TABLE 2: COMPARISON OF THE ET1 LEVEL IN PLASMA AND VOLUMES OF EDEMA AND HEMATOMA BETWEEN TWO GROUPS (MEAN \pm SD)

\begin{tabular}{lcccc}
\hline Group & Time points & Plasma ET1 $(\mathrm{ng} / \mathrm{L})$ & Edema volume $(\mathrm{mL})$ & Hematoma volume $(\mathrm{mL})$ \\
\hline Control group $(\mathrm{n}=63)$ & Before treatment & $129.66 \pm 11.64$ & $6.87 \pm 2.09$ & $20.46 \pm 3.72$ \\
& After treatment & $93.54 \pm 8.69^{\mathrm{a}}$ & $4.54 \pm 1.39^{\mathrm{a}}$ & $15.36 \pm 3.90^{\mathrm{a}}$ \\
$\mathrm{t}$ & & 19.0814 & 7.0912 & 7.2494 \\
$\mathrm{a} \mathrm{p}$ & & $<0.01$ & $<0.01$ & $<0.01$ \\
Observation group & Before treatment & $130.52 \pm 11.43$ & $6.54 \pm 2.41$ & $21.57 \pm 3.97$ \\
$(\mathrm{n}=63)$ & After treatment & $73.21 \pm 8.34^{\mathrm{ab}}$ & $2.10 \pm 1.24^{\mathrm{ab}}$ & $9.87 \pm 3.53^{\mathrm{ab}}$ \\
$\mathrm{t}$ & & 31.0811 & 12.5211 & 16.8721 \\
$\mathrm{ap}$ & $<0.01$ & $<0.01$ & $<0.01$ \\
$\mathrm{t}$ & & 12.9499 & 9.9861 & 7.9952 \\
$\mathrm{~b} p$ & $<0.01$ & $<0.01$ & $<0.01$ \\
\hline
\end{tabular}

Note: ${ }^{\mathrm{p}} \mathrm{p}<0.01$ represents the significant difference of intragroup comparison; ${ }^{\mathrm{b}} \mathrm{P}<0.01$ represents the significant difference of observation group vs control.

TABLE 3: COMPARISON OF THE NIHSS SCORES BETWEEN TWO GROUPS (MEAN \pm SD)

\begin{tabular}{lccc}
\hline \multirow{2}{*}{ Group } & Before treatment & \multicolumn{2}{c}{ After treatment } \\
\cline { 3 - 4 } & $22.92 \pm 6.74$ & After two w & After four w \\
\hline Control group $(\mathrm{n}=63)$ & $21.66 \pm 7.33$ & $16.59 \pm 5.12$ & $11.49 \pm 1.80$ \\
Observation group $(\mathrm{n}=63)$ & 0.9732 & $12.43 \pm 4.07^{\mathrm{a}}$ & $7.52 \pm 1.84^{\mathrm{a}}$ \\
$\mathrm{t}$ & $>0.05$ & 4.8958 & 11.9122 \\
$\mathrm{ap}$ & $<0.01$ & $<0.01$ \\
\hline
\end{tabular}

Note: ${ }^{a} p<0.05$ represents the significant difference with control group.

In cerebral hemorrhage, the major pathological injuries include the post-hemorrhagic mechanical suppression of hematoma on the surrounding brain tissues, injury of hemorrhage to brain tissues and the aggravation of inflammation induced by the activated inflammatory cells after hematoma, which pose severe threatens to the life of patients ${ }^{[6]}$. Patients, however, usually suffer the deterioration of $\mathrm{HICH}$, since the attack of hemorrhage activates the polymorphonuclear leukocyte that increases the autophagic activity and generation of free radicals, thereby inducing the peroxidation of lipid, injury to the vascular endothelial cells and increase in the membrane permeability ${ }^{[7]}$. Thus, elimination of free radicals is of great significance in treatment of $\mathrm{HICH}$. Evidence has shown that edaravone in combination with dihydropyridine calcium ion antagonist can improve the efficacy, with shrinkage in the volumes of edema and hematoma and recovery of nerve functions ${ }^{[8]}$.

In this study, we found that the total effectiveness rate in the control group was $71.44 \%$, significantly lower than the observation group, and after treatment, the level of ET1 in serum, edema and hematoma volumes were all lowered as compared with the levels before treatment, while the decreases in the observation group were more evident. No significant difference was found in comparison of the NIHSS scores before treatment, while the scores in the observation group, after two and four $\mathrm{w}$ of treatment, were all higher than those in the control group, which was similar to the findings of Junqiang ${ }^{[9]}$, demonstrating that on the basis of the regular comprehensive treatment, edaravone in combination with dihydropyridine calcium ion antagonist can decrease the level of ET1 in serum, with the shrinkage in the volumes of hematoma and edema, which benefit the recovery of nerve function with precise efficacy.

Edaravone, as one of the eliminator of free radicals, can eliminate the cytotoxic hydroxide radicals in brain tissues, which can decrease the concentration of hydroxide radicals and curb the brain edema ${ }^{[10]}$. In addition, edaravone can increase the expression of nerve growth factors to protect the brain tissues, thereby benefiting the recovery of nerve function. ET1, mainly distributed in the cardiovascular and nervous system, has a potent ability of vasocontraction ${ }^{[11]}$. $\mathrm{HICH}$ patients, due to the calcium overload in nerve cells, suffer from the brain injury, which, plus the ischemia and hypoxia, can induce the massive generation of ET1 in the damaged vascular endothelial cells, thus 
aggravating the disease. Nimodipine, as a kind of dihydropyridine calcium ion antagonist with apotent lipid solubility, can pass through the brain-blood barrier to block the L-type calcium channel and the calcium influx. In addition, nimodipine can activate the $\mathrm{Ca}^{2+}-$ ATPase to augment the elimination of cytoplastic calcium, thereby mitigating the calcium overload and reduce the synthesis and release of ET in the brain tissues and vascular endothelial cells, thereby relaxing the smooth muscle, increasing the blood flow, enhancing the vessel dilation and the tolerance of brain tissues to the ischemia ${ }^{[12]}$. Moreover, nimodipine can improve the secondary brain ischemia after hemorrhage, which is conducive to the nervous dysfunction caused by the severe cerebrovascular spasm. Meanwhile, nimodipine can decrease the content of Antibodies to aquaporin-4 (AQP4) to mitigate the brain edema ${ }^{[13]}$. With a potent selectivity on the brain tissues, nimodipine can avoid the pial veins, which can guarantee the regular modulation of blood flow and dilation of vessels in brain tissues, without blood loss. Thus, edaravone in combination with nimodipine can enhance the elimination of free radicals, and mitigate the calcium overload in the nerve cells, which can improve the vasospasm and inhibit the progression of disease ${ }^{[14]}$.

Furthermore, combined medication of edaravone and nimodipine can improve the blood cycle in the brain by resolve or absorb the hemorrhage-induced brain hematoma to mitigate the suppression on the surrounding tissues, promote the recovery of nerve functions and improve the prognosis. However, further work is required to illustrate the effect of many factors on the efficacy in this study.

Thus, combined medication of edaravone and dihydropyridine calcium ion antagonist can benefit the patients with promising efficacy and improvement in nervous function. This strategy is worthy of being promoted in clinical practice.

\section{REFERENCES}

1. Zhang Y, Yang Y, Zhang GZ, Gao M, Ge GZ, Wang QQ, et al. Stereotactic Administration of Edaravone Ameliorates Collagenase-Induced Intracerebral Hemorrhage in Rat. CNS Neurosci Ther 2016;22(10): 824-35.
2. Aguilar MI, Brott TG. Update in Intracerebral Hemorrhage. Neurohospitalist 2011;1:148-59.

3. Mittal MK, LacKamp A. Intracerebral Hemorrhage: Peri hemorrhagic Edema and Secondary Hematoma Expansion: From Bench Work to Ongoing Controversies. Front Neurol 2016;7:210.

4. Qiuju Li, Zhiling Ji, Kun Li. Effect of combination of parenteral edaravone and nimodipine on ischemic cerebral injury following cerebral hemorrhage. Tropical J Pharma Res 2018;17(5):955-60.

5. Zhang XL, Zheng SL, Dong FR, Wang ZM. Nimodipine Improves Regional Cerebral Blood Flow and Suppresses Inflammatory Factors in the Hippocampus of Rats with Vascular Dementia. J Int Med Res 2012;40(3):1036-45.

6. Aronowski J, Zhao X. Molecular pathophysiology of cerebral hemorrhage: Secondary brain injury. Stroke 2011;42(6):17816.

7. Mracsko E, Veltkamp R. Neuroinflammation after intracerebral hemorrhage. Front Cell Neurosci 2014;8:388.

8. Kikuchi K, Takeshige N, Miura N, Morimoto Y, Ito T, Tancharoen S, et al. Beyond free radical scavenging: Beneficial effects of edaravone (Radicut) in various diseases. Exp Ther Med 2012;3(1):3-8.

9. Qiuju Li, Zhiling Ji, Kun Li. Effect of Edaravone Combined with Nimodipine on Hypertensive Intracerebral Hemorrhage. Health for Everyone 2017;(24):76.

10. Wang GH, Jiang ZL, Li YC, Li X, Shi H, Gao YQ, et al. Free-Radical Scavenger Edaravone Treatment Confers Neuroprotection Against Traumatic Brain Injury in Rats. J Neurotrauma 2011;28(10): 2123-34.

11. Kikuchi K, Tancharoen S, Takeshige N, Yoshitomi M, Morioka M, Murai Y, et al. The Efficacy of Edaravone (Radicut), a Free Radical Scavenger, for Cardiovascular Disease. Int J Mol Sci 2013;14(7):13909-30.

12. Wang F, Cao Y, Ma L, Pei H, Rausch WD, Li H. Dysfunction of Cerebrovascular Endothelial Cells: Prelude to Vascular Dementia. Front Aging Neurosci 2018;10:376.

13. Ren C, Gao J, Xu J, Xu H, Liu G. The Nimodipine-Sparing Effect of Perioperative Dexmedetomidine Infusion During Aneurysmal Subarachnoid Hemorrhage: A Prospective, Randomized, Controlled Trial. Front Pharmacol 2019;10:858

14. Wang G, Su J, Li L, Feng J, Shi L, He W, Liu Y. Edaravone alleviates hypoxia-acidosis/reoxygenation-induced neuronal injury by activating ERK1/2. Neurosci Lett 2013;543:72-7.

This is an open access article distributed under the terms of the Creative Commons Attribution-NonCommercial-ShareAlike 3.0 License, which allows others to remix, tweak, and build upon the work non-commercially, as long as the author is credited and the new creations are licensed under the identical terms

\footnotetext{
This article was originally published in a special issue, "Trends in Therapeutic Management of Various Clinical Conditions II" Indian J Pharm Sci 2021:83(2)Spl issue;175-178
} 